\title{
A ATENCÃO DA ENFERMAGEM NA SAÚDE DA CRIANÇA: REVISÃO INTEGRATIVA DA LITERATURA
}

Mariana Marques Viera

Maria Carolina Ortiz Whitaker

Angela Aparecida Costa

Janaina Mery Ribeiro ${ }^{l}$

\section{RESUMO}

$\mathrm{Na}$ infância ocorrem modificações, que influenciam o desenvolvimento e a fase adulta. Por isso, merecem atenção dos serviços de saúde. A assistência deve ser integral, buscando atender as necessidades da criança em diferentes momentos de sua vida. Pensando nisso, questiona-se: qual a preocupação da enfermagem com a saúde da criança na atenção básica à saúde? O objetivo do trabalho foi identificar o conhecimento científico produzido atualmente, sobre a preocupação da enfermagem com a saúde da criança na atenção básica à saúde. Para tanto, foi realizada uma revisão integrativa da literatura com o estabelecimento das palavras-chave para responder à pergunta norteadora: Criança, Atenção Primária à Saúde e Enfermagem; identificadas no DeCS, e no MeSH, utilizadas em Criança, Atenção Primária à Saúde e Enfermagem; identificadas no DeCS, e no MeSH, utilizadas em
português e inglês. Após a delimitação dos objetivos e questionamentos a serem respondidos, houve uma busca sistemática e integral na Biblioteca Virtual em Saúde, que contempla as bases de dados: LILACS, IBECS, MEDLINE, Biblioteca Cochrane, SciELO e BDENF, de artigos em português e inglês, publicados entre 2003 e 2013. A amostra foi de 18 artigos após a aplicação dos critérios e inclusão e exclusão, como utilizar apenas textos com resumo disponível, que contemplavam a temática estabelecida. Foram excluídos aqueles direcionados a adolescentes, com temáticas específicas, com eixo de estudo centrado em patologias. Trabalhos publicados anteriormente a 2003 não fizeram parte da seleção. A amostra foi dividida em quatro temáticas principais: 'Avaliação e descrição da estrutura da atenção básica para atender a crianças', 'A atenção ao crescimento e desenvolvimento infantil', 'Atenção em puericultura a crianças menores de dois anos de idade' e 'A estratégia de Atenção Integrada às Doenças Prevalentes na Infância (AIDPI)' O estudo revelou preocupação da enfermagem com o cuidado em puericultura e com a saúde de crianças de até dois anos de idade, fator positivo para a qualidade do cuidado e redução da mortalidade infantil. Para os descritores
utilizados não foram encontrados trabalhos com crianças em fase escolar e observou-se a existência de poucas publicações que tratam sobre a articulação entre os serviços. Concluindo, há preocupação da enfermagem com a saúde da criança, porém existem assuntos a serem abordados pela enfermagem para o aprimoramento da qualidade assistencial.

Palavras-chave: Criança; Atenção Primária à Saúde; Enfermagem. 


\section{NURSING CARE IN CHILD HEALTH: \\ AN INTEGRATIVE REVIEW OF THE LITERATURE}

\section{ABSTRACT}

Changes occur in childhood that influence the development and adulthood. Therefore, they deserve attention of health services. Assistance should be integral, seeking to meet the child's needs at different times in his life. Considering this, the question is: what is the concern of nursing with the health of children in primary health care? The objective was to identify the scientific knowledge currently produced on the concern of nursing with the health of children in primary health care. To this end, an integrative literature review was carried out with the establishment of the keywords to answer the guiding question: Child, Primary Health Care and Nursing, identified in DeCS and $\mathrm{MeSH}$, used in Portuguese and English. After the establishment of the objectives and questions anstematic and comprehensiv which includes the following databases: LILACS, IBECS, MEDLINE, Cochrane Library, SciELO and BDENF, articles in Portuguese and English, published between 2003 and 2013. The sample consisted of 18 articles after the application of inclusion and exclusion criteria and, using only texts with available abstracts, that contemplated the established theme. The ones directed to adolescents with specific themes, with study axis centered on pathologies, were excluded. Works published previously to 2003 were not part of the selection. The sample was divided into four main themes: 'Evaluation and description of primary care structure to assist children', 'Attention to child growth and development', 'Warning in childcare to children under two years of age' and 'The Integrated Management for Childhood Illness (IMCI) '. The study showed nursing concern in child care and in the health of children under two years of age, a positive factor for the quality of care and the reduction of child mortality. Considering the selected keywords, works with schoolchildren were not found. Also, it was observed the existence of few publications that deal with the relationship among the services. In conclusion, there is concern of nursing with children health, but there are issues to be addressed by nursing to improve the quality of care.

Keywords: Child; Primary Health Care; Nursing.

\section{INTRODUÇÃO}

Ainfância é uma fase marcada por mudanças decorrentes do crescimento e desenvolvimento do indivíduo. É influenciada pelas variações do ambiente físico, familiar e social em que a criança está inserida. Os acontecimentos na vida da criança, bem como seu desenvolvimento e crescimento, exercerão grande influência na fase adulta, tornando esta fase, um período de grande relevância no ciclo vital. Ao considerarmos as faixas etárias dos usuários dos serviços de saúde, a maior demanda é compreendida pelos extremos de idade. Por se tratar de um período dinâmico, é assim um momento que requer atenção e cuidado dos profissionais e serviços de saúde (SILVA, 2012).

Portanto, torna-se indispensável, que haja atenção e planejamento dos serviços de saúde, políticas públicas de saúde e legislação, voltada às crianças (TOMASI et al., 2009). Entende-se por criança, indivíduos até 12 anos de idade, incompletos; segundo o Estatuto da Criança e do Adolescente, regulamentado pela Lei 8.069/90. Estes, com direito à proteção integral, garantida pela família, comunidade e sociedade em gera (BRASIL, 1990)

Segundo dados do último censo populaciona do Instituto Brasileiro de Geografia e Estatística (IBGE), apesar do envelhecimento populacional, uma grande parcela da população é composta por criancas e adolescentes, dos mais de 190 milhões de habitantes do Brasil, cerca de 29 m de habitantes do Brasil, cerca de 29 milhões são crianças com idade entre 0 e 9 anos e 35 milhões, crianças e jovens com idade entre 10 e 19 anos, totalizando cerca de 63 milhões de crianças e jovens. Percentualmente, em 2010 o censo concluiu que $24,08 \%$ da população brasileira é composta po crianças entre 0 e 14 anos (BRASIL, 2010).

A atenção à saúde da criança no Brasil é marcada pelas influências políticas, econômicas e sociais do país. No ano de 1984, o Ministério da Saúde lançou o Programa, Assistência Integral à Saúde da Criança: Ações Básicas (PAISC), que orientou as práticas de saúde acerca do aleitamento materno, orientações alimentares para o desmame, o controle de doenças diarreicas e respiratórias na infância, controle de imunizações e do crescimento e desenvolvimento infantil. Este programa marcou o utilizando atividades de baixa complexidade e custo (FIGUEIREDO; MELLO, 2003).

Direcionado pelo modelo de atenção à saúde e proposto pela Conferência de Alma-Ata, pautado na promoção da saúde e atenção integral ao individuo, surgiu no Brasil o modelo único de saúde, cuja proposta de atenção primária tem a função de acolher e oferecer resolubilidade da maioria dos agravos trazidos pela população (TOMASI et al., 2009).

O Sistema Único de Saúde trata a saúde como direito de todos os cidadãos, sem distinção de crença, etnia ou situação econômica, e dever do Estado (BRSAIL, 1988). Regulamentado pelas Leis Orgânicas, $\mathrm{N}^{\circ}$ 8.142/90 e No 8.080/90, garante condições de bem-estar físico, mental e social, para determinar saúde e a participação social nas decisões de políticas de saúde que atendam à demanda populacional (BRASIL, 1990).

A difícil situação do grupo infantil, marcada por altas taxas de mortalidade e ausência de ações efetivas para prevenção, promoção e reabilitação da saúde, direcionou que a Organização Mundial de Saúde (OMS), junto das Nações Unidas em Favor da Infância (Unicef), desenvolvessem a estratégia Infância (AIDPI), que foi adotada pelo Ministério da Saúde em 1996, com o objetivo de caracterizar e integrar as doenças de maior prevalência na infância e adolescência, capacitando principalmente os profissionais de saúde para uma redução da morbi-mortalidade infantil, por causas evitáveis, foi pensado para modificar a situação da saúde da criança (BRASIL, 2002).

Diante da adoção da estratégia AIDPI, houve uma redução da mortalidade infantil, de $6 \%$ em 10 anos, entre 1994 e 2004, segundo a estimativa realizada em 2004, a primeira, que foi realizada 8 anos após a completa implementação da estratégia 
AIDPI pelo governo brasileiro. Além disso, houve redução das hospitalizações, em um terço do total anterior (PINA et al., 2009).

Apesar dos avanços e redução das taxas de mortalidade infantil é fundamental a manutenção e ampliação da assistência à saúde da criança na atenção básica e intervenções de enfermagem prioritárias voltadas para: promoção do nascimento saudável, acompanhamento do recém-nascido de risco, acompanhamento do crescimento e desenvolvimento, seguimento da imunização, promoção do aleitamento materno e alimentação saudável, atenção aos distúrbios nutricionais e abordagem das doenças respiratórias e infecciosas que visam cuidado integral, vigilância e promoção da saúde (MELLO; TONETE; SILVA, 2009).

$\mathrm{O}$ enfermeiro que atua em serviços primários de saúde desenvolve ações de promoção, proteça prevenção e educação em saúde, bem como, cuidados para tratamento e reabilitação, no âmbito individual e coletivo (MONTEIRO et al., 2011).

Dessa forma, entendemos que, aprender sobre os cuidados de enfermagem à criança na Atenção Básica à Saúde é reconhecer a assistência e as práticas desenvolvidas para essa clientela, assim questionamos: Qual o conhecimento científico produzido, acerca da atuação da enfermagem, junto às crianças, na atenção básica à saúde?

\section{OBJETIVO}

O objetivo deste estudo foi identificar qual o conhecimento científico produzido atualmente, pela enfermagem, sobre sua preocupação com a saúde da criança na atenção básica à saúde e; levanta quais ações são desenvolvidas pelo enfermeiro para crianças na atenção básica à saúde.

\section{METODOLOGIA}

Para alcançar o objetivo do trabalho, o método escolhido foi uma revisão integrativa da literatura, método de pesquisa que permite síntese do

conhecimento produzido sobre um determinado assunto e aponta lacunas a serem preenchidas com a realização de novas pesquisas. É um método de grande valia para a enfermagem, pois permite aos profissionais, que nem sempre têm tempo de ler diversas pesquisas sobre um mesmo tema, estudar em um único trabalho uma síntese do conhecimento, isso contribui para a qualidade da prática profissional (MENDES; SILVEIRA; GALVÃO, 2008)

Segundo Torraco (2005); A revisão integrativa da literatura é uma forma de pesquisa que analisa, critica e sintetiza a literatura representada em um tópico de uma maneira integrada, de tal forma que novas perspectivas do tema são geradas.

A elaboração da revisão integrativa necessita da delimitação do objetivo específico do trabalho, formulação dos questionamentos a serem respondidos; realização da busca para a coleta do maior número possível de pesquisas primárias que serão utilizadas de acordo com os critérios de inclusão e exclusão pré-determinados. Para tanto são delimitadas etapas como, identificação do tema escolhido (elaboração da pergunta norteadora, estabelecimento das palavras chaves a serem utilizadas e dos critérios para inclusão ou exclusão dos artigos encontrados); seleção dos artigos; definição das informações a serem retiradas destes, como objetivos, metodologia e conclusões; assim como a análise, discussão e interpretaç̃o dos resultados e sintese do conhecimento adquirido (SOUZA; SILVA; CARVALHO, 2010).

Compreendendo a importância de ações de enfermagem na atenção primária à saúde, que priorizem a promoção a saúde infantil, a prevenção de agravos e a identificação precoce de sinais e sintomas, foi elaborada a pergunta norteadora deste estudo: Qual o conhecimento científico produzido, acerca da preocupação da enfermagem com a saúde da criança, na atenção básica à saúde?

Os critérios de inclusão foram: os artigos deveriam contemplar a saude da criança na assistência prestada na atenção básica, como temática principal; texto em português ou inglês, publicados entre janeiro de 2003 e junho de 2013; publicações com resumos disponíveis na Biblioteca Virtual em Saúde (BVS), que contempla as seguintes bases de dados, relacionadas às Ciências da Saúde em Geral: LILACS (Literatura LatinoAmericana e do Caribe em Ciências da Saúde), IBECS (Índice Bibliográfico Espanhol de Ciências da Saúde), MEDLINE (Literatura Internacional em Ciências da Saúde), Biblioteca Cochrane, SciELO (Scientific Eletronic Library Online) e BDENF (Base de Dados de Enfermagem).

Foram excluídos os trabalhos que não contemplavam a temática estabelecida, assim como aqueles direcionados a adolescentes ou com temáticas específicas, com eixo de estudo centrado em patologias; artigos que não apresentavam resumo também não foram incluídos no referencial teórico do trabalho. Trabalhos publicados anteriormente a 2003 não fizeram parte da seleção.

Quadro 1. Descrição dos artigos encontrados segundo base de dados e critérios de inclusão/exclusão

\begin{tabular}{|c|c|c|}
\hline \multirow{2}{*}{ Descritores } & \multicolumn{2}{|c|}{$\begin{array}{r}\text { Enfermagem/Nursing (and) Criança/Child (and) Atenção Primária } \\
\text { àde/Primary Health Care. }\end{array}$} \\
\hline Base de Dados & Busca Inicial & Critérios de Inclusão/Exclusão \\
\hline LILACS & 248 & 5 \\
\hline IBCS & 34 & 0 \\
\hline MEDLINE & 106 & 2 \\
\hline Biblioteca Cochrane & 3 & 0 \\
\hline SciELO & 367 & 8 \\
\hline BDENF & 193 & 18 \\
\hline Total & 951 & 3 \\
\hline
\end{tabular}

Fonte: Arquivo Pessoal. Araraquara, 2014.

As palavras-chave utilizadas foram criança (child), atenção primária à saúde (primary health care) e enfermagem (nursing), extraídas dos descritores no DeCS (Descritores em Ciências da Saúde), e do MeSH (Medical Subject Headings).

Para a seleção dos artigos, foi realizado o cruzamento das palavras-chave, resultando o total de 951 artigos. Na primeira seleção foram encontrados 214 artigos com resumos disponíveis. Após a leitura dos títulos e resumos para a identificação dos artigos, 132, destes, foram estes, foram excluídos 64 títulos, devido à repetição dos artigos nas bases de dados e publicação anterior a 2003. Apos leitura detalhada e atenta, a amostra . cruzamento das palavras chaves e a quantidade de artigos retiradas de cada base de dados, ante e após a aplicação dos critérios de inclusão e exclusão: 


\section{RESULTADOS E DISCUSSÃO}

Ao realizar a análise dos 18 artigos selecionados, foi possível observar que 17 deles foram realizados por enfermeiros. A maioria dos artigos da amostra, foi escrita no ano de 2011, totalizando 5 artigos. No ano de 2012, foram escritos 4, nos anos de 2003, 2009 e 2010, duas publicações por ano. Para 2005, 2008 e 2013, foi encontrada uma publicação para cada ano, que atendesse ao tema desejado. Nos anos não citados, nenhum texto correspondeu aos descritores e ao tema delimitado. Quanto, aos países de nenhum texto correspondeu aos descritores e ao tema delimitado. Quanto, aos países de

origem, para esta amostra foi delimitado o idioma,

apenas artigos em português e inglês entrariam na seleção, dentre essas duas origens, a maioria das publicações foi brasileira, totalizando 16 textos, uma publicação norte-americana, e, uma publicação britânica.

Em relação às características do tipo de estudo, 17 possuíam abordagem metodológica qualitativa, um deles, abordagem qualitativa-quantitativa. Entre os estudos revisados, dez desenvolveram trabalhos descritivos, dois estudos transversais descritivos, um avaliativo, um observacional, uma revisão da literatura e três basead cúlise de ou analise de dados. O quadro abaixo faz uma breve síntese das publicações que obedeceram aos critérios

Quadro 2. Descrição dos artigos que compuseram a amostra do estudo - Araraquara, 2013.

\begin{tabular}{|c|c|c|}
\hline Identificação & $\begin{array}{l}\text { Tipo de } \\
\text { Pesquisa }\end{array}$ & Principais Resultados \\
\hline $\begin{array}{l}\text { 1. Atenção integrada } \\
\text { às doenças prevalentes } \\
\text { na infância (ADIPI) na } \\
\text { prática de enfermeiros } \\
\text { egressos da USP. } \\
\text { Higuchi C. H., et al; } \\
\text { 2011. SP. }\end{array}$ & $\begin{array}{c}\text { Qualitativa } \\
\text { Estudo de } \\
\text { Campo }\end{array}$ & $\begin{array}{l}\text { Apesar de considerarem fundamental a } \\
\text { estratégia AIDPI na saúde da criança, os } \\
\text { egressos afirmaram que não a utilizam de forma } \\
\text { sistematizada, como preconizado, devido a } \\
\text { algumas dificuldades. Porém mesmo restrita, } \\
\text { a estratégia permite ao enfermeiro prestar uma } \\
\text { assistência integral e integrada, o que justifica } \\
\text { sua abordagem durante a graduação. }\end{array}$ \\
\hline $\begin{array}{l}\text { 2. A formação do } \\
\text { enfermeiro e a estratégia } \\
\text { atenção integrada às } \\
\text { doenças prevalentes na } \\
\text { infância. } \\
\text { Veríssimo M. R., et al; } \\
\text { 2003. SP. }\end{array}$ & $\begin{array}{l}\text { Qualitativa } \\
\text { Revisão de } \\
\text { Literatura }\end{array}$ & $\begin{array}{l}\text { O artigo reuniu aspectos históricos sobre a } \\
\text { estratégia AIDPI, fundamentais à prática do } \\
\text { enfermeiro, principalmente aquele atuante em } \\
\text { saúde infantil. Assim, pode contribuir para } \\
\text { uma melhor atuação do enfermeiro na prática } \\
\text { assistencial. }\end{array}$ \\
\hline
\end{tabular}

3. Contribuições da estratégia Atenção Integrada às Doenças prevalentes na Infância

ao acolhimento de

crianças menores de cinco anos.

Pina J. C., et al; 2009. SP.

4. Puericultura em

Enfermagem: perfil e principais problemas encontrados em crianças menores de um ano. Gauterio D. P., et al; 2012. DF.

\section{A avaliação da atenção} à saúde de crianças com menos de um ano de idade na Atenção Primária.

Furtado M. C. C., et al; 2013. SP.

6. Avaliação da atenção ao recém-nascido

na articulação entre maternidade e rede básica de saúde.

Furtado M. C. C., et al; 2010. SP.
O estudo revelou que a estratégia AIDIPI contribui para a prática profissional, situações prioritárias puderam ser Qualitativa identificadas rapidamente, bem como Estudo de outros problemas. Apesar de alguns Campo aspectos ainda precisarem ser corrigidos, o estudo mostrou potencial reprodução da estratégia em outras unidades de saúde, por favorecer a qualidade assistencial.

Qualina prevenção de agravos e promoção da saúde. E também afirma que é preciso dar condições

de acesso aos usuários.

O estudo considera de extrema importância Quantitativa a investigação do cuidado prestado às criança Estudo de nas unidades de saúde. Ele revelou que há Erande vínculo entre as mães e a equipe, nas Campo unidades acompanhadas, e a importância dessa relação.

O estudo avaliou uma estratégia de articulação entre a maternidade e a rede básica de saúde. Qualitativa Ele apontou que apesar de algumas dificuldades Estudo de de operacionalização, essa ação é fundamental à Campo criança, por garantir a integralidade. $O$ trabalho foi importante para avaliação e melhoria das ações. 


\begin{tabular}{|c|c|c|}
\hline $\begin{array}{l}\text { 7. Developments in } \\
\text { child health surveilance } \\
\text { programmes. } \\
\text { Taylor, C.; 2005. London }\end{array}$ & $\begin{array}{c}\text { Qualitativa } \\
\text { Revisão de } \\
\text { Literatura }\end{array}$ & $\begin{array}{l}\text { Após avaliar as diretrizes produzidas e } \\
\text { publicadas por um Grupo de Trabalho em } \\
\text { Saúde infantil, o autor concluiu que as ações } \\
\text { de vigilância devem incentivar a promoção } \\
\text { de saúde e prevenção primária, a desenvolver } \\
\text { ações curativas. }\end{array}$ \\
\hline $\begin{array}{l}\text { 8. Health counselling: } \\
\text { parental-oriented health } \\
\text { dialogue - na innovation } \\
\text { for child. } \\
\text { Golsäter M., et al; } 2009 . \\
\quad \text { London. }\end{array}$ & $\begin{array}{l}\text { Qualitativa } \\
\text { Estudo de } \\
\text { Campo }\end{array}$ & $\begin{array}{l}\text { O estudo avaliou um instrumento de } \\
\text { acompanhamento da saúde da criança, utilizado } \\
\text { por enfermeiros britânicos. Ele constatou que } \\
\text { esse instrumento direciona a consulta e torna } \\
\text { mais fácil a compreensão dos enfermeiros sobre } \\
\text { o processo saúde-doença, qualificando assim, } \\
\text { a assistência. }\end{array}$ \\
\hline $\begin{array}{l}\text { 9. Avaliação da estrutura } \\
\text { destinada à consulta de } \\
\text { enfermagem à criança na } \\
\text { atenção básica. } \\
\text { Saparolli E. C. L., Adami, } \\
\text { N. P.; 2010. SP. }\end{array}$ & $\begin{array}{l}\text { Quali- } \\
\text { quantitativa } \\
\text { Estudo de } \\
\text { Campo }\end{array}$ & $\begin{array}{l}\text { A estrutura destinada ao atendimento infantil } \\
\text { na atenção básica apresentou-se satisfatória } \\
\text { frente à sua utilização. A qualificação da } \\
\text { enfermeira, por sua vez, foi avaliada como } \\
\text { apropriada. Pode se dizer então que há } \\
\text { qualidade na assistência prestada ao considerar } \\
\text { essas duas vertentes. }\end{array}$ \\
\hline $\begin{array}{l}\text { 10. Modelando a } \\
\text { integralidade do cuidado } \\
\text { à criança na atenção } \\
\text { básica de saúde. } \\
\text { Sousa, F. G. M., et al; } \\
\text { 2010. RS. }\end{array}$ & $\begin{array}{l}\text { Qualitativa } \\
\text { Estudo de } \\
\text { Campo }\end{array}$ & $\begin{array}{l}\text { A integralidade é um conjunto de práticas } \\
\text { que correspondem à necessidade de } \\
\text { saúde da criança e sua família. Isso } \\
\text { impõe grandes desafios e inquietações } \\
\text { aos profissionais. O trabalho propõe a } \\
\text { re-criação de modos de cuidados, aliando } \\
\text { saber técnico e subjetivo. }\end{array}$ \\
\hline
\end{tabular}

\begin{tabular}{|c|c|c|}
\hline $\begin{array}{l}\text { 11. Condições limitadoras } \\
\text { para a integralidade do } \\
\text { cuidado à criança na } \\
\text { atenção básica de saúde. } \\
\text { Sousa F. G. M., et al; } \\
\text { 2011. SC. }\end{array}$ & $\begin{array}{c}\text { Qualitativa } \\
\text { Estudo de } \\
\text { Campo }\end{array}$ & $\begin{array}{l}\text { Após ouvir estrategicamente profissionais e } \\
\text { usuários, o estudo revelou que vários fatores } \\
\text { são limitantes para a prática da integralidade } \\
\text { na assistência, a falta de insumos, inadequação } \\
\text { da estrutura, entre outros. Isso reflete em um } \\
\text { baixo impacto da ABS na saúde da criança e } \\
\text { é explicada pela desarticulação entre os níveis } \\
\text { de atenção. }\end{array}$ \\
\hline $\begin{array}{l}\text { 12. O trabalho de } \\
\text { enfermagem na alta de } \\
\text { crianças hospitalizadas. } \\
\text { Silva, R. V. G. O., } \\
\text { Ramos, F. R. S.; } 2011 . \\
\text { RS. }\end{array}$ & $\begin{array}{c}\text { Qualitativa } \\
\text { Estudo de } \\
\text { Campo }\end{array}$ & $\begin{array}{l}\text { O estudo apontou que o enfermeiro consegue } \\
\text { praticar a integralidade, durante a permanência } \\
\text { da criança no hospital, porém no segmento } \\
\text { pós-alta verificou-se uma fragilidade na } \\
\text { integralidade por praticamente não existir } \\
\text { uma articulação entre os serviços. Contatando- } \\
\text { se assim uma lacuna entre os dois níveis de } \\
\text { assistência. }\end{array}$ \\
\hline $\begin{array}{l}\text { 13. Qualificando o } \\
\text { cuidado à criança na } \\
\text { Atenção primária à } \\
\text { Saúde. } \\
\text { Sousa, F. G. M., } \\
\text { Erdmann, A. L.; } 2012 . \\
\text { DF. }\end{array}$ & $\begin{array}{c}\text { Qualitativa } \\
\text { Estudo de } \\
\text { Campo }\end{array}$ & $\begin{array}{l}\text { O presente estudo indicou que o conceito de } \\
\text { saúde como ausência de doença apenas, já está } \\
\text { ultrapassado e que o cuidado à criança sustenta- } \\
\text { se a partir de um saber compartilhado, a partir } \\
\text { disso buscou-se compreender como ocorre o } \\
\text { cuidado na ABS. }\end{array}$ \\
\hline $\begin{array}{l}\text { 14. Competências da } \\
\text { enfermeira para a atenção } \\
\text { à criança na rede básica } \\
\text { de saúde. } \\
\text { Silva, A. M.; 2012. RS. }\end{array}$ & $\begin{array}{l}\text { Quali- } \\
\text { quantitativa } \\
\text { Estudo de } \\
\text { Campo }\end{array}$ & $\begin{array}{l}\text { O estudo mostrou que a maioria das competências } \\
\text { da enfermagem em relação à saúde da criança } \\
\text { envolve também a família, o que está de acordo } \\
\text { com as políticas e programas vigentes. O estudo } \\
\text { dará subsidio á pratica profissional. }\end{array}$ \\
\hline
\end{tabular}




\begin{tabular}{|c|c|c|}
\hline $\begin{array}{l}\text { 15. A prática de } \\
\text { enfermagem na atenção } \\
\text { à saúde da criança em } \\
\text { unidade básica de saúde. } \\
\text { Figueiredo, G. L.; Mello, } \\
\text { D. F.; 2003. SP }\end{array}$ & $\begin{array}{l}\text { Qualitativa } \\
\text { Estudo de } \\
\text { Campo }\end{array}$ & $\begin{array}{l}\text { O estudo buscou identificaro acompanhamento } \\
\text { do crescimento e desenvolvimento infantil em } \\
\text { uma UBS. Foi possível perceber que ações } \\
\text { importantes e orientações pertinentes a esta } \\
\text { fase são fornecidas aos pais, porém de forma } \\
\text { fragmentada e sem vinculação. }\end{array}$ \\
\hline $\begin{array}{l}\text { 16. A expressão da } \\
\text { autonomia do enfermeiro } \\
\text { no acompanhamento } \\
\text { do crescimento e } \\
\text { desenvolvimento da } \\
\text { criança. } \\
\text { Monteiro, A. I., et al; } \\
\text { 2011. RJ. }\end{array}$ & $\begin{array}{l}\text { Qualitativa } \\
\text { Estudo de } \\
\text { Campo }\end{array}$ & $\begin{array}{l}\text { Este estudo descreveu a autonomia do } \\
\text { enfermeiro no processo de planejamento e } \\
\text { implementação da proposta de acompanhamento } \\
\text { coletivo do crescimento e desenvolvimento } \\
\text { infantil. Autonomia esta expressa na capacidade } \\
\text { de avaliação e identificação de problemas e } \\
\text { criação de soluções. Denota-se a importância } \\
\text { de fortalecer o desenvolvimento de trabalhos } \\
\text { educativos voltados à coletividade. }\end{array}$ \\
\hline $\begin{array}{l}\text { 17. A enfermagem } \\
\text { e o fazer coletivo: } \\
\text { Acompanhando } \\
\text { o crescimento e } \\
\text { desenvolvimento da } \\
\text { criança. } \\
\text { Monteiro, A. I., et al; } \\
\quad \text { 2011. CE. }\end{array}$ & $\begin{array}{l}\text { Qualitativa } \\
\text { Estudo de } \\
\text { Campo }\end{array}$ & $\begin{array}{l}\text { O trabalho descreveu o processo de introdução } \\
\text { do acompanhamento e desenvolvimento } \\
\text { coletivo por enfermeiros, acompanhamento } \\
\text { este que norteia as ações básicas de saúde } \\
\text { voltadas às crianças. O trabalho concluiu que } \\
\text { por meio da educação é que os profissionais } \\
\text { se fortalecem cientificamente e desenvolvem } \\
\text { ações qualificadas. }\end{array}$ \\
\hline $\begin{array}{l}\text { 18. Desenvolvimento } \\
\text { infantil: concordância entre } \\
\text { a caderneta de saúde e o } \\
\text { manual para vigilância do } \\
\text { desenvolvimento infantil. } \\
\text { Oliveira, L. L., et al; 2012. SP. }\end{array}$ & $\begin{array}{l}\text { Qualitativa } \\
\text { Estudo de } \\
\text { Campo }\end{array}$ & $\begin{array}{l}\text { O objetivo deste trabalho foi identificar } \\
\text { uma concordância entre a caderneta de } \\
\text { saúde da criança e o Manual para Vigilância } \\
\text { do desenvolvimento infantil e após análise } \\
\text { percebeu-se que há baixa concordância entre } \\
\text { esses parâmetros. }\end{array}$ \\
\hline
\end{tabular}

Fonte: Arquivo Pessoal. Araraquara, 2013.

106 • Revista Uniara

Touniara

Volume $18, n^{\circ} 1$, julho de 2015
A análise das publicações selecionadas permitiu a identificação de quatro temáticas principais, avaliação e descrição da estrutura da atenção básica para atender a crianças, atenção ao crescimento e desenvolvimento infantil, atenção em puericultura e a criancas menores de dois anos de idade e, a estratégia AIDPI

\section{Atenção em puericultura e a crianças} menores de dois anos de idade

A assistência à saúde da criança modificou-se ao longo dos anos, em decorrência às modificações do perfil epidemiológico da população. Atualmente, o conjunto de enfermidades que acometem a população já é outro e a descoberta de tecnologias e incorporação de atividades profissionais também contribuíram para a mudança na assistência

A divisão da saúde da criança em faixas etárias facilita a compreensão e a identificação de agravos muitas vezes comuns a uma mesma faixa etária. As características inerentes a cada faixa etária auxiliam o diagnóstico e orientam o modo de prestar o cuidado à criança (VERISSIMO et al., 2003).

Muitos agravos, facilmente controláveis após os primeiros anos de vida, são fatais para esta faixa etária, se não diagnosticados precocemente. A avaliação constante e regular do peso e crescimento e vital para o desenvolvimento posterior da criança. O primeiro ano é o momento fundamental para o bom desenvolvimento das etapas que virão no ciclo vital. É por isso que essa fase merece um cuidado especial, na atenção primária à saúde, que deve ser iniciada na saída da maternidade. Portanto, os serviços devem articular-se para que não haja lacunas no atendimento, destacando-se de form integral. Vale ressaltar que nessa, assim como em toda fase da vida da criança, a família deve ser considerada no atendimento à criança, uma vez que é relevante para a formação do contexto na qual esta se encontra (FURTADO et al., 2013).

Para Gauterio, Irala e Cesar-Vaz. (2012), que realizou um estudo qualitativo sobre o perfil da população menor de um ano atendida por enfermeiros em puericultura numa UBS em Rio Grande - RS, há a necessidade de sensibilizar a população sobre a importância do acompanhamento em puericultura para que agravos sejam prevenidos e para o desenvolvimento de ações de promoção de saúde aos pequenos cidadãos.

A unidade básica de saúde representa para essas mães, muitas vezes inexperientes, uma referência para o cuidado de seu filho. Elas frequentam a unidade para consultas de rotina e para situações de emergência. A proximidade da mãe com a unidade começa durante a gestação e isso facilita o acesso e confiança na equipe de saúde (FURTADO et al., 2013).

\section{A estratégia AIDPI}

A estratégia de Atenção Integrada às Doenças Prevalentes na Infância (AIDPI) surgiu no momento histórico em que as maiores causas de mortalidade infantil, até os 5 anos de idade, eram desnutrição, doenças diarreicas e doenças respiratórias, agravos estes que, se identificados precocemente $\mathrm{e}$ devidamente tratados, não causariam tanto impacto na saúde da criança como mostravam as estatísticas. A ideia surge de uma iniciativa da Pastoral da Criança, inicialmente no nordeste do país - região onde os índices de mortalidade eram, e ainda são, maiores -, de incentivar praticas de higiene multimistura, para combater a desnutrição. A finalidade era diminuir significativamente, em curto período de tempo, os índices de mortalidade infantil. Para isso, foi proposta uma nova abordagem, pela ONU, juntamente com a UNICEF, abordagem esta caracterizada por ser simultânea e integrada das doenças de maior prevalência, ao contrário da abordagem tradicional (BRASIL, 2002).

Pina et al. (2009), descreveu em seu trabalho, o acolhimento estruturado pela estratégia AIDPI em uma USF, demonstrando que a promoção da saúde infantil vai além da preocupação com a diminuição 
compromisso pela qualidade de vida da criança. Eé nesse contexto que está inserida a estratégia AIDPI a qual engloba os principais fatores que afetam a saúde da criança de uma forma sistematizada, contemplando um conjunto de condições básicas de vida, como alimentação, moradia, saneamento básico, educação, lazer, entre outras.

Higuchi et al. (2011), realizou um estudo qualitativo acerca da introdução da estratégia AIDPI na prática de enfermeiros egressos da USP, observou as potencialidades e limitações do desenvolvimento dessas ações; entre elas, a estratégia de atenção às doenças prevalentes na infância, que quando incorporada ao atendimento da atenção primária à saúde, pode diminuir não só os índices de mortalidade infantil, mas também as altas taxas de morbidade e, consequentemente, de internações. Para tanto, o enfermeiro da atenção básica deve ter a qualificação para essa estratégia desde a graduação. O AIDPI possibilita uma visão global da criança, abrangendo o contexto social familiar que envolve sua vida. Integra atividades teóricas e práticas com a finalidade de oferecer uma atenção integral à criança.

Outros projetos marcaram a preocupação das instituições com a saúde da criança, a começar pelo PAISC, publicado pela primeira vez em 1984 e que, já naquele momento, instituía ações básicas, discutidas até hoje, para a promoção da saúde da criança. Apesar do esforço profissional, porém, a saúde da criança continuava sendo um desafio à saúde nacional (VERISSIMO et al., 2003)

Segundo Higuchi (2011); seguindo a ordem cronológica e espacial, a estratégia foi implantada primeiramente no nordeste, região de maior urgência de ações relativas à saúde da criança, devido aos índices de mortalidade infantil, no ano de 1996. Seis anos depois, em 2002, todos os estados brasileiros contavam com profissionais capacitados para desenvolver estas ações na atenção primária.

Complementar a isto, Veríssimo (2003) descreveu em seu trabalho a introdução da AIDPI na formação dos enfermeiros, afirmando que a
Atenção Primária é caracterizada por trabalhar desde ações preventivas até ações paliativas, e pela grande necessidade da criação de vínculo entre profissional e paciente. Nesse sentido, é mais adequado classificar os problemas identificados à explanar diagnósticos clínicos.

\section{Atenção ao crescimento
e desenvolvimento infantil}

O acompanhamento do crescimento e desenvolvimento infantil dentro dos parâmetros de normalidade e atendendo às peculiaridades de cada criança é a maneira mais fácil de identificar agravos precocemente. Este deve ser sustentado por um profissional qualificado, que conhece as características normais para poder identifica possiveis anormalidades sutis, que, por muitas vezes, podem passar despercebidas até se tornarem mais graves e irreversíveis. O acompanhamento deve ser regular, e isso só é possível quando há relação interpessoal entre o profissional e os responsáveis pela criança, relação esta possibilitada pelo grau de atenção destinado ao atendimento. Para se alcançar a integralidade no cuidado a criança, é necessário que haja uma dedicação profissional para um atendimento completo, que enxerga a criança como um ser biopsicossocial, e não apenas a queixa apresentada, num atendimento fragmentado (OLIVEIRA et al., 2012)

Para Monteiro et al. (2011), acompanhar o crescimento e desenvolvimento infantil é fundamental à saúde da criança, pois fornece subsídios à avaliação da morbimortalidade. Atualmente, esse acompanhamento acontece, muitas vezes, através de consultas individuais, pautadas no modelo biomédico, ou seja, na vigência e necessidade de ações curativas. Isso resulta na falta de entendimento dos usuários acerca da importância de ações de promoção de saúde e acompanhamento periódico das crianças.

$\mathrm{Na}$ década de 80 , numa tentativa de reorganização do sistema e diminuição da morbimortalidade infantil, o governo federal em conjunto com as secretarias estaduais e municipais e o Ministério da Saúde, criou o programa "Assistência Integral à Saúde da Criança", a fim de criar vínculo entre paciente e profissional, incentivando o acompanhamento sistemático do crescimento e desenvolvimento infantil (FIGUEIREDO; MELO, 2003).

A reorientação do modelo de assistência está pautada, de forma que a criança seja vista em seu contexto biopsicossocial, com ações voltadas à promoção de saúde, prevenção de agravos, reabilitação ou recuperação da saúde, em todos os níveis de atenção, de maneira integrada e multiprofissional (MONTEIRO et al., 2011)

A nova proposta apresenta uma rede de serviços hierarquizada de acordo com a complexidade, sendo a ABS entrada preferencial do usuário no sistema, cuidados básicos de saúde de forma integral, e níveis secundário e terciário, com maior nível de tecnologia envolvida, recebendo casos mais complexos, referenciados. Assim, oferece uma proposta de atendimento universal, com interação dos setores e atenção multiprofissional, em que cada saber tem sua importância durante o processo de assistência à saúde (FIGUEIREDO; MELO, 2003)

Para Monteiro et al. (2008), na vivência prática, há a busca por um modelo que atenda às necessidades de saúde dos usuários dentro da própria $\mathrm{ABS}$. Como estratégia, temos a educação em saúde, que desenvolve a criticidade dos usuários, além de considerar seu próprio conhecimento sobre o serviço e todo o seu processo saúde-doença.

Em síntese, temos que a infância é um período único da vida do indivíduo e sabe-se que exerce grande influência sobre a fase adulta. Essa etapa é fundamental à todas as subsequentes. $\mathrm{O}$ cuidado à criança envolve preocupações com o desenvolvimento, que se não forem corretamente abordadas, afetarão a fase adulta e, consequentemente, a sociedade. Além disso, a oportunidade de orientação, incentivando a promoção de saúde, é ampla durante esse período, e muitos comportamentos conseguem ser modificados mais facilmente (MOREIRA; GOLDANI, 2010).
Para Prado (2005), as crianças demandam maior atenção dos sistemas de saúde pelo fato de apresentarem maior suscetibilidade, devido à dependencia e rápida alteração nas fases de atividades do setor primário, se estas forem, de fato, efetivas, a necessidade de hospitalização e as consequências trazidas por ela serão menores. Ademais, haverá a liberação de leitos para outros casos de necessidade.

A busca pela integralidade traz consigo uma abordagem holística da criança, ultrapassando a visão biológica do processo saúde-doença imprimindo um caráter emocional na assistência prestada (ROCHA et al., 1997).

Em relação à Rede Básica, se a assistência desse setor for resolutiva, haverá menor necessidade erianca para outros niveis de atenção, poupando recursos humanos, materiais e tecnológicos, além de manter o sistema para procedimentos realmente necessários (FRANCO; MAGALHÃES, 2004)

Em relação às publicações acadêmicas, para Rocha et al. (1997), a enfermagem tem produzido muito acerca da integralidade, conceito que é amplamente discutido em ambientes científicos. A prática deste conceito, porém, ainda é insipiente.

\section{Avaliação e descrição da estrutura da}

atenção básica pa

A atenção básica pode ser entendida como um grupo de ações que tem como objetivo a promoção de saúde, prevenção de agravos e reestabelecimento da saúde, tanto na esfera individual, quanto coletiva. Porém, a resolubilidade deste nível de atenção deve ser tamanha, que ações não devem ser pautadas apenas como curativas e individuais. E para que a atenção à saúde seja efetiva, a unidade deve conhecer sua população, suas singularidades e os determinantes do processo saúde-doença em sua região (SAPAROLLI; ADAMI, 2010).

A estrutura física, qualificação profissional e quantidade de profissionais envolvidos são 
algumas características pontuais para uma atenção integral à criança. Para que haja qualidade do serviço prestado, é necessário que o profissional disponha de uma estrutura adequada, abrangendo atendimento da demanda populacional, assim como deve haver profissionais capacitados, qualificados e disponíveis em número adequado, para não ocorrer sobrecarga de trabalho e redução do rendimento (SAPAROLLI; ADAMI, 2010).

Em relação à qualificação profissional, Taylor et al. (2004), realizou um estudo que avaliava as diretrizes propostas por um Grupo de Trabalho em Saúde Britânico sobre a saúde da criança. Após o estudo, ele conclui que as recomendações de um programa de vigilância contínua da saúde infantil deve ter maior ênfase em ações de promoção de saúde e prevenção primária à incentivar ações curativas.

Na saúde coletiva, a autonomia do enfermeiro torna-se mais expressiva através do atendimento direto à população, durante consultas de enfermagem, ações e atividades de promoção de saúde em caráter individual e coletivo. Quando o enfermeiro se aproxima de questões burocráticas, ele, indiretamente, se afasta de ações de cuidado e, consequentemente, há grande perda de autonomia Além disso, a consulta de enfermagem é um momento único para a vinculação do profissional com o paciente e sua família, principalmente na saúde da criança, período que deve ser valorizado (MONTEIRO et al., 2011).

Sousa et al. (2011), após realizar um estudo de campo para compreender quais fatores limitam a prática assistencial de cuidado integral a criança, percebeu que questões políticas, institucionais e gerenciais, além de fatores profissionais e pessoais, interferem no modo como é prestada a assistência limitando sua prática integral.

Frente a isso, para que o enfermeiro possa assumi este papel e para que esta prática tenha qualidade, os serviços de saúde devem dispor de estrutura adequada, como áreas físicas, instrumentos de trabalho, materiais e outros equipamentos, além da

capacitação profissional e número de enfermeiros adequados ao desenvolvimento de ações como essa, diariamente (SAPARROLI; ADAMI, 2010).

Sousa et al. (2010); desenvolveu um estudo com objetivo de compreender como é prestada a assistência à criança na atenção básica a saúde, na perspectiva da integralidade, o modo como o profissional aborda os pais e sua qualificação faz toda diferença para que a integralidade seja alcançada e mantida, ou não. O profissional deve estar apto a aproximar-se dos pais através do trabalho em equipe e realizar práticas intersertoriais, aliando o saber técnico ao subjetivo.

Golsäter et al. (2009), após realizar um estudo que avaliou um instrumento utilizado por enfermeiros britânicos para acompanhamento da criança, a curva de saúde constatou que, por meio da utilização de um instrumento de coleta de dados que direcione os profissionais para uma consulta de enfermagem completa, o enfermeiro consegue melhor diálogo com os pais, compreendendo o processo saúdedoença destes como um todo, não apenas avaliando os agravos que trazem a criança à unidade.

A articulação entre os níveis de atenção também é fundamental para que a integralidade no atendimento à criança seja atingida. Existem fatores na prática profissional, porém, que limitam a operacionalização de uma assistência integral, como falta de estrutura e profissionais, conflitos, insegurança, entre outras coisas (MONTEIRO et al., 2008)

Silva; Ramos (2011), após desenvolver um estudo sobre a articulação entre a atenção básica a saúde e o setor terciário, constatou a existência de uma lacuna entre esses dois níveis de atenção. Esse fator limita a atuação do enfermeiro da atenção básica, uma vez que este, muitas vezes, não identifica a criança em alta hospitalar para captá-la. Isso remete a necessidade de uma reorganização do processo de trabalho nesses dois níveis.

O cuidado, principalmente à criança, é complexo multidimensional, e sofre influências de diversas formas em que as ações são desenvolvidas. A proposta pautada em redes de serviços, tornando a assistência de fato integral, apresenta este modelo que exige do sistema, profissionais, equipe instituições e unidades de saúde que se organizem de modo a produzir uma assistência multi, inter e transdisciplinar, na qual todos os integrantes da equipe de saúde contribuem para a sua qualidade (SOUSA; ERDMANN, 2012)

Incluir a família a este cuidado também é fundamental para que os objetivos propostos sejam alcançados, uma vez que a criança não tem autonomia para tomar decisões a respeito de seu tratamento e, muitas vezes, é altamente dependente dos pais. Isso é competência do enfermeiro de atenção báscia a saúde, que deve responsabilizar os pais e trazê-los para a unidade de saúde para cuidarem de seus filhos (SILVA, 2012).

\section{CONCLUSÕES}

Com base nos descritores utilizados para a coleta dos artigos e da análise dos mesmos, foi possíve observar que há uma preocupação por parte dos profissionais de enfermagem com a saúde da criança na atenção básica de saúde.

Em relação ao ano de publicação, a maioria

foi publicada após o ano de 2010, totalizando 11 artigos, os demais foram entre os anos de 2003 a 2009.

Para os descritores utilizados, o maior número de publicações encontradas, foi escrita por enfermeiros. participaram das equipes multiprofissionais produziram sobre a saúde da criança, adicionando uma contribuição fundamental para a qualificação da equipe e promoção da assistência.

Diante do tema e dos descritores utilizados, pudemos observar, também, que a maior preocupação tem sido com as crianças entre 0 e 5 anos, com ênfase para os menores de 1 ano. Em relação à saúde do escolar, fase compreendida entre 6 e 12 anos, uma entre as diversas classificações existentes para essa faixa etária, Fujimori; Ohara (2009) levanta uma questão: será que há preocupação da enfermagem com esta fase do desenvolvimento? $\mathrm{O}$ que se produz acerca desta preocupação?

Ressaltamos que não foi possível identificar abordagens, referente ao quantitativo de recursos humanos envolvidos na atenção à saúde da criança necessidade de futuras publicações que busquem descrer sobre a quantidade real e a necessária, para a implementação de ações como exemplo, a consulta de enfermagem.

Os temas encontrados foram divididos em quatro grupos de assuntos, sendo que o primeiro tema abordado foi, a atenção em puericultura e a crianças menores de dois anos, por ser uma faixa etária muito específica e diferente das posteriores, merecendo uma atenção dos serviços em decorrência da instabilidade deste grupo etário. E também, por ser um período de conhecimento e adaptação entre mãe estreito, pois nessa faixa etária muitos agravos são fatais, se não descobertos precocemente.

O segundo tema descreveu sobre importância que teve a estratégia AIDPI na qualificação de enfermeiros atuantes na atenção básica a saúde e, consequentemente, na redução das taxas de morbimortalidade infantil, agregando às crianças melhor qualidade de vida e condições de saúde, e o conhecimento de enfermeiros recém-formados sobre esta estratégia de atenção, já que as doenças trazidas pelo AIDPI não correspondem mais à maioria, mas que ainda devem receber uma atenção especial pela sua gravidade.

A atenção ao crescimento e desenvolvimento infantil, foi o terceiro tema levantado nessa revisão e ressaltou a importância do acompanhamento contínuo destes como um instrumento de vigilância e detecção precoce de doenças ou agravos e assim assegurar às crianças saúde e um futuro com boas perspectivas de vida. Acompanhamento que deve sempre ser feito na por ações básicas de saúde, mesmo na ausência da doença, para desenvolver promoção de saúde e prevenção de agravos.

Além destes temas, encontramos a influência da estrutura da atenção básica na assistência prestada 
às crianças. É na atenção básica a saúde que devemos encontrar a resolutividade de pelo menos $80 \%$ das necessidades de saúde da população. A estrutura física disponível, qualificação profissional, quantidade e envolvimento de profissionais são algumas características pontuais para que a atenção a saúde da criança seja de fato integral. Os estudos nos revelaram que a autonomia do enfermeiro de atenção básica mostra-se mais expressiva quando este se distancia de questões burocráticas e aproxima-se do atendimento à sua população. Os quatro temas encontrados para os de utilizados, descrevem vertentes desenvolvidas na atenção básica a saúde para melhorar a saúde da criança no cenário atual. Reintegramos a necessidades da ampliação de estudos que foquem a importância e a descrição de como vem se consolidando na prática a comunicação entre serviços de atenção primária, secundária e terciária de saúde para compreendermos como tem sido a evidencia da integralidade do cuidado; assim como a realização de estudos que demonstrem como tem sido a inserção do cuidado de crianças em condições crônicas de saúde na atenção básica.

\section{REFERÊNCIAS}

BRASIL, AIDPI: Atenção Integrada às Doenças Prevalentes na Infância. Ministério da Saúde. Brasília. 2002. Disponível em: <http://bvsms. saude.gov.br/bvs/publicacoes /aidpi_modulo_1. pdf $>$ Acesso em: 06 Jun. 2013.

BRASIL, Atenção Humanizada ao Recémnascido de Baixo Peso - Método Canguru. Ministério da Saúde. Brasília. 2002. Disponível em: <http://bvsms.saude.gov.br/bvs/publicacoes metodo canguru manual tecnico_2ed.pdf $>$ Acesso em: 08 Ago. 2013.

BRASIL, Contagem Populacional. Ministério do Planejamento, Orçamento e Gestão. Instituto Brasileiro de Geografia e Estatística. 2010. Disponível em: < http://www.ibge.gov.br/home/ estatistica/populacao/censo2010/default.shtm> Acesso em: 28 Ago. 13.

BRASIL, Estatuto da Criança e do Adolescente. Governo Federal. Brasília. 1990 181p. Disponível em: <http://www.planalto gov.br/ccivil_03/leis/18069.htm> Acesso em: 10 Jun. 2013. [1].

BRASIL, Lei 8.080. Governo Federal. Brasília. 1990. Disponível em: < http://www planalto gov. $\mathrm{br} /$ ccivil 03/leis/18080.htm> Acesso em: 12 Jun. 2013. [2].

BRASIL, Lei 8.142. Governo Federal. Brasília. 1990. Disponível em: <http://www.planalto.gov. br/ccivil 03/leis/18142.htm> Acesso em: 09 Jun 2013. [3].

FIGUEIREDO, G. L. A.; MELLO. D. F. - A prática de enfermagem na atenção à saúde da criança em Unidade Básica de Saúde. Rev. LatinoAm. Enfermagem. Ribeirão Preto. v. 11. n. 4. p. 544 a 551. 2003. Disponível em: <http://www. scielo.br/scielo.?scriptsciarttext\&p $>$ Acesso em: 15 Ago. 2013.

FRANCO, T. B.; MAGALHÃES, H. M. J. - Integralidade na assistência à saúde: a organização das linhas do cuidado. São Paulo: Hucitec, 2004. $2^{\mathrm{a}}$ ed.

FUJIMORI, E. (Org.); OHARA, C. V. S. (Org) - Enfermagem e a saúde da criança na atenção básica. Barueri: Manole. 2009.

FURTADO, M. C. C.; BRAZ, J. C.; PINA, J. C.; MELLO, D. F.; LIMA, R. A. G. - A avaliação da atenção à saúde de crianças com menos de um ano de idade na Atenção Primária. Revista Latino-Am. Enfermagem. Ribeirão Preto. v. 21 n. 2. p. 554 a 561. 2013. Disponível em: $<\mathrm{http}: / /$ www.scielo.br/ scielo.php?pid=S0104-11690554\&scriptt $>$ Acesso em: 08 Jun. 2013
FURTADO, M. C. C.; MELLO, D. F.; PARADA, C. M. G. L.; PINTO, I. C.; REIS, M. C. G.; SCOCHI, C. G. S. - Avaliação da atenção ao recémnascido na articulação entre maternidade e rede básica de saúde. Rev. Eletr. Enf. [S.1.]. v. 12. n. 4. p. 640 a 646. 2010. Disponível em: < http://www. revistas.ufg.br/index.php/fen/article/view/7625> Acesso em: 03 Ago. 2013.

GAUTERIO, D. P; IRALA, D. A., CEZAR-

VAZ, M. R. - Puericultura em Enfermagem: perfil e principais problemas encontrados em crianças menores deumano. RevistaBrasileiradeEnfermagem. Brasília. v. 65. n. 3. p. 508 a 513. 2012. Disponível em: <http://www.scielo.br/scielo.php?script=sci arttext\&pid=S0034-71672012000300017 $>$ Acesso em: 25 Jun. 2013

GOLSÄTER, M.; ENSKÄR, K.; LINGFORS, H.; SINDENVALL, B. - Health counseling: parental-oriented health dialogue - an innovation for child health nurses. Journal of Child Health Care. Londres. v. 13. n. 1. p. 75 a 88. 2009. Disponível em:< http://www.ncbi nlm.nih.gov/pubmed/19240192> Acesso em: 03 Jun. 2013.

HIGUCHI, C. H.; FUJIMORI, E.; CURSINO, E. G.; CHIESA, A. M.; VERISSIMO, M. D. L. O R.; MELLO, D. F. - Atenção Integrada às Doenças Prevalentes na Infância na prática de enfermeiros egressos da USP. Revista Gaúcha de Enfermagem. PortoAlegre. v. 32.n.2.p. 241 a247.2011. Disponível em: $<$ http://www.scielo.br/scielo.php?script=sci arttext\&pid=S1983-14472011000200005 $>$ Acesso em: 05 Ago. 2013.

MENDES, K. D. S.; SILVEIRA, R. C. C. P. GALVÃO, C. M. - Revisão integrativa: método de pesquisa para a incorporação de evidências na saúde e na enfermagem. Texto Contexto Enfermagem. Florianópolis. v. 17. n. 4. p. 758 a 764. 2008 Disponível em: < http://www.scielo.br/pdf/tce/ v17n4/18.pdf $>$ Acessado em: 20/04/2013.
MELLO, D. F.; TONETE, V. L. P.; SILVA, M. A. I. - A Atenção Básica á Saúde da Criança. In: FUJIMORI, E. (Org.); OHARA, C. V. S. (Org). Enfermagem e a saúde da criança na atenção básica. Barueri: Manole. 2009. p. 44-60.

MONTEIRO, A. I.; MACEDO, I. P.; SANTOS A. D. B.; ARAÚJO, W. M. - A enfermagem e o fazer coletivo: acompanhando o crescimento e o desenvolvimento da criança. Rev. Rene. Fortaleza. v. 12.n. 1. p. 73 a 80. 2011. Disponível em: < http: www revistarene ufc br/vol12n1 pdf/a10v12n1. pdf> Acesso em: 03 Mai. 2013.

MONTEIRO, A. I.; SANTOS, A. D. B.; MACEDO, I. P.; GURGEL, P. K. F.;

MENDES, K. D. S.; SILVEIRA, R. C. C. P.; GALVÃO C. M - Revisão integrativa: método de pesquisa para a incorporação de evidências na saúde e na enfermagem. Texto Contexto Enfermagem. Florianópolis. v. 17. n. 4. p. 758 a 764. 2008. Disponível em: <http://www.scielo.br/pdf/tce/ v17n4/18.pdf $>$ Acesso em: 12 Jun. 2013.

MONTEIRO, A. I.; SANTOS, A. D. B.; MACEDO,I.P.; GURGEL,P.K.F.; CAVALCANTE, J.M.P. - A expressão da autonomia do enfermeiro no acompanhamento de crescimento e desenvolvimento da criança. Rev. Enferm. UERJ. Rio de Janeiro. v. 19. n. 3. p. 426 a 431. 2011. Disponível em: $<$ http:// www.facenf.uerj.br/v19n3/v19n3a15.pdf $>$ Acesso em: 20 Abr. 2013.

MOREIRA, M. E. L.; GOLDANI, M. Z. - A criança é o pai do homem: novos desafios para a área de saude da criança. Ciência e Saúde Coletiva Rio de Janeiro. v. 15. n. 2. p. 321 a 327. 2010 Disponível em: <http://www.arca.fiocruz.br/ bitstream/icict/1737/1/A\%20crian\%C3\%A7a\%20 \%C3\%A9\%20o\%20pai\%20do\%20homem.pdf $>$ Acesso em: 20 Jun. 2013

OLIVEIRA, L. L.; COTA, V. M. R.; 
S.; PIMENTA, A. F.; LEMOS, S. M. A. Desenvolvimento infantil: concordância entre a caderneta de saúde da criança e o manual para vigilância do desenvolvimento infantil. Rev. Paulista de Pediatria. São Paulo. v. 30. n. 4. p. 479 a 485. 2012. Disponível em: < http://www.scielo.br/scielo.php?pid=S0103$05822012000400004 \&$ script $=$ sci_arttext $>$ Acesso em: 03 Jun. 2013

PINA, J. C.; MELLO, D. F.; MISHIMA, S. M.; LUNARDELO, R. - Contribuições da Estratégia Atenção Integrada às Doenças Prevalentes na Infância ao acolhimento de crianças menores de cinco anos. Acta Paulista de Enfermagem. São Paulo. v. 22. n. 2. p. 142 a 148. 2009. Disponível em: < http://www. scielo.br/pdf/ape/v22n2/a05v22n2> Acesso em: 04 Mai. 2013.

PRADO, S. R. L. A. - Integralidade: Um estudo a partir da atenção básica à saúde da criança em modelos assistenciais distintos. Tese (Doutorado em Enfermagem) - Escola de Enfermagem. Universidade de São Paulo. São Paulo. 2005. Disponível em: < http://www.teses. usp.br/teses/disponiveis/7/7136/tde-27012006105705/pt-br.php> Acesso em: 04 Jun. 2013.

ROCHA, S. M. M.; LIMA, R. A. G.; SCOCHI, C. G. S. - Assistência integral à saúde da criança no Brasil: implicações para o ensino e a prática da enfermagem pediátrica. Saúde e Sociedade. São Paulo. 1997. v. 6. n. 1. Disponível em: <http:// www.scielo.br/pdf/sausoc/v6n1/04.pdf $>$ Acesso em: 01 Set. 2014.

SAPAROLLI, E. C. L.; ADAMI, N. P. Avaliação da estrutura destinada à consulta de enfermagem à criança na atenção básica. Rev. Esc. Enferm. USP. São Paulo. v. 44. n. 1. p. 92 a 98. 2010. Disponível em: <http://www. scielo.br/scielo.phpscriptsciarttextpid $=001>$ Acesso em: 01 Set. 2014.

SILVA, A. M. - Competências da enfermeira para a atenção à criança na Rede Básica de Saúde. Tese (Mestrado em Enfermagem) Universidade Federal do Rio Grande do Sul. Escola de Enfermagem. Programa de PósGraduação em Enfermagem. Porto Alegre. 2012. Disponível em: < http://www.lume. ufrgs.br/handle/10183/55427> Acesso em: 04 Jun. 2013.

SILVA, R. V. G. O.; RAMOS, F. R. S. - O trabalho da enfermagem na alta de crianças hospitalizadas: articulação da atenção hospitalar e básica. Rev. Gaúcha de Enfermagem. Porto Alegre. v. 32. n. 2. p. 309 a 315. 2011. Disponível em: <http://www.scielo.br/scielo.php?pid=S1983$14472011000200014 \&$ script=sci arttext $>$ Acesso em: 02 Set. 2014.

SOUSA, F. G. M.; ERDMANN,A. L.; MOCHEL, E. G. - Condições Limitadoras para a Integralidade do Cuidado à Criança na Atenção Básica de Saúde. Texto Contexto Enfermagem. Florianópolis v. 20 n. 1. p. 263 a 271. 2011. Disponível em: $<$ http://www.scielo.br/scielo.php?pid=S0104 $07072011000500033 \&$ script $=$ sci_arttext $>$ Acesso em: 01 Ago. 2014

SOUSA, F. G. M.; ERDMANN, A. L.; MOCHEL, E. G. - Modelando a integralidade do cuidado à criança na Atenção Básica de Saúde. Rev. Gaúcha de Enfermagem. Porto Alegre. v. 31 n. 4. p. 701 a 707. 2010. Disponível em: $<\mathrm{http}: / /$ www.scielo.br/scielo.php? script=sci

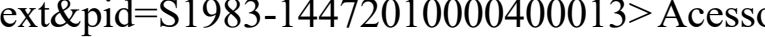
em: 20 Set. 2014

SOUSA, F. G. M.; ERDMANN, A. L. Qualificando o cuidado à criança na Atenção Primária de Saúde. Rev. Brasileira de Enfermagem. Brasília. v. 65. n. 5. p. 795 a 802. 2012. Disponível em: $<$ http://www.scielo.br/scielo.php?pid=S0034 $71672012000500012 \&$ script $=$ sci arttext $>$ Acesso em: 30 Set. 2013.
SOUSA, M. T.; SILVA, M. D.; CARVALHO, R. - Revisão integrativa: o que é e como fazer Einstein. São Paulo. v. 8. n. 1. p. 102 a 106. 2010 Disponível em: <http://scielo.com.br $>$ Acesso em: 18 Jun. 2013.

TAYLOR, C. - Developments in child. health surveillance programmes. Nursing Times. Londres. v. 101. n. 27. p. 32. 2005. Disponível em: $<$ http://www.nursingtimes.net/nursing-practice/ specialisms/public-health/developments-in-childhealth-surveillance-programmes/203779.article> Acesso em: 02 Ago. 2013.

TOMASI, E.; FACCHINI, L. A.; THUMÉ, E.; PICCINI, R. X.; OSORIO, A.; SILVEIRA, D. S.; SIQUEIRA, F. V.; TEIXEIRA, V. A.; DILÉLIO, A S.; MAIA, M. F. S. - Características da utilização de serviços de Atenção Básica à Saúde nas regiões Sul e Nordeste: diferenças por modelo de atenção. Ciência \& Saúde Coletiva. São Paulo. v. 16. n. 11. p. 4395 a 4404. 2009. Disponível em: < http://www. scielo.br/pdf/csc/v16n11/a12v16n11.pdf > Acesso em: 05 Ago. 2014.

TORRACO, R. J. - Writing Integrative Reviews: Guidelines and Examples.

Academy of Human Resource Development. Nebrasca. v. 4. n. 3. p. 356 a 367. 2005. Disponível em: <http://www.sagepub.com/gray/Website $\% 20$ material/Journals/hrd torraco.pdf $>$ Acesso em: 25 Nov. 2013.

VERISSIMO, M. D. L. O. R.; MELLO, D F.; BERTOLOZZI, M. R.; CHIESA, A. M.; SIGAUD, C. H. S.; FUJIMORI, E.; LIMA, R. A. G. - A Formação do Enfermeiro e a estratégia de Atenção Integrada às Doenças Prevalente na Infância. Revista Brasileira de Enfermagem. Brasília. v. 56. n. 4. p. 396 a 400. 2003. Disponível em: < http://www.scielo.br/ pdf/reben/v56n4/a18v56n4.pdf $>$ Acesso em: 04 Nov. 2013. 